\title{
The sRNA MicC downregulates hilD translation to control the SPI1 T3SS in Salmonella enterica serovar Typhimurium
}

\author{
Fatih Cakar ${ }^{1}$, Yekaterina A. Golubeva ${ }^{1}$, Carin K. Vanderpool ${ }^{1}$ and James M. Slauch ${ }^{1}$ \\ ${ }^{1}$ Department of Microbiology \\ University of Illinois at Urbana-Champaign
}

Running Title: MicC regulates hilD translation

\#Corresponding Author. E-mail: slauch@illinois.edu

Department of Microbiology

University of Illinois

B102 Chemical and Life Sciences Laboratory

601 S Goodwin Avenue

Urbana Illinois, 61801

Phone: (217) 244-1956 


\section{ABSTRACT}

Salmonella enterica serovar Typhimurium invades the intestinal epithelium and induces inflammatory diarrhea using the Salmonella pathogenicity island 1 (SPI1) type III secretion system (T3SS). Expression of the SPI1 T3SS is controlled by three AraC-like regulators, HilD, HilC and RtsA, which form a feed-forward regulatory loop that leads to activation of $h i l A$, encoding the main transcriptional regulator of the T3SS structural genes. This complex system is affected by numerous regulatory proteins and environmental signals, many of which act at the level of hilD mRNA translation or HilD protein function. Here, we show that the sRNA MicC blocks translation of the hilD mRNA by base pairing near the ribosome binding site. This binding blocks translation but does not induce degradation of the hilD message. Our data indicate that micC is transcriptionally activated by SlyA, and SlyA feeds into the SPI1 regulatory network solely through MicC. Transcription of $m i c C$ is negatively regulated by the OmpR/EnvZ two-component system, but this regulation is dependent on SlyA. OmpR/EnvZ control SPI1 expression partially through MicC, but also affect expression through other mechanisms. MicC-mediated regulation plays a role during infection, as evidenced by an increase in Salmonella fitness in the intestine in the micC deletion mutant that is dependent on the SPI1 T3SS. These results further elucidate the complex regulatory network controlling SPI1 expression and add to the list of sRNAs that control this primary virulence factor.

\section{IMPORTANCE}

19 The Salmonella SPI1 T3SS is the primary virulence factor required for causing intestinal disease and initiating systemic infection. The system is regulated in response to a large variety of environmental and physiological factors such that the T3SS is expressed at only the appropriate

22 time and place in the host during infection. Here we show how the sRNA MicC affects expression

23 of the system. This work adds to our detailed mechanistic studies aimed at a complete

24 understanding of the regulatory circuit.

\section{INTRODUCTION}

Salmonella enterica serovar Typhimurium causes inflammatory diarrhea and potentially life-threatening systemic infection, with an estimated global burden of 95 million cases per year

28 world-wide (1). Upon oral ingestion, Salmonella transits through the stomach to reach the distal

29 ileum of the small intestine, the initial site of colonization $(2,3)$. Salmonella invades the intestinal

30 epithelium of the host and induces inflammatory diarrhea using the Salmonella pathogenicity 31 island 1 (SPI1) type III secretion system (T3SS), a needle-like structure that injects effector 32 proteins into the host cell cytosol (4). 
The SPI1 T3SS is controlled by three AraC-like transcriptional regulators, HilD, HilC and RtsA, which constitute a complex feed-forward regulatory loop, each activating transcription of the hilD, hilC, and $r$ tsA genes, as well as activating hilA, encoding the transcriptional regulator of T3SS structural genes (Fig. 1)(5). This system is controlled in response to numerous regulatory proteins and environmental signals, many of which act at the level of hilD mRNA translation or stability, or HilD protein activity $(6,7)$. This includes regulation by a number of small RNAs (8).

Small RNAs (sRNAs), generally 50 to $450 \mathrm{bp}$ in length, are increasingly recognized as critical regulators of gene expression (9). Over 300 sRNAs are expressed in Salmonella. They play important roles in the regulation of envelope stress responses, metabolism, and virulence. However, the function of most of these sRNAs is unknown or only partially characterized. Many sRNAs control gene expression by imperfect base-pairing with an mRNA near the ribosome binding site (RBS), mediated by the RNA chaperone Hfq $(9,10)$. This blocks access to the $30 \mathrm{~S}$ ribosomal subunit, inhibiting translation initiation. In some cases, this also leads to RNaseEmediated degradation of the message (11).

The OmpR/EnvZ two-component regulatory system was originally characterized as the regulator of the $\mathrm{OmpF}$ and $\mathrm{OmpC}$ porins in response to changes in osmolarity (12). OmpR/EnvZ is now understood to be a global regulator of virulence in Salmonella, activating both the SPI2 and SPI1 type three secretion systems, despite the fact that these systems are primarily required in different niches (13-15). The transmembrane histidine kinase EnvZ autophosphorylates and transfers a phosphoryl group to the response regulator OmpR. At low concentrations of OmpR$\mathrm{P}, o m p F$ is activated, while at high concentrations of $O m p R-P, o m p F$ is repressed and ompC is activated (16). The sRNA MicF is transcribed upstream and antisense to ompC by OmpR. MicF, one of the first sRNAs identified $(17,18)$, base pairs with the ompF mRNA to block translation and facilitate the transition from producing $\mathrm{OmpF}$ to $\mathrm{OmpC}$ porin in high osmolarity. More recently, the MicC sRNA was identified and characterized as a regulator of the outer membrane porin OmpC in E. coli that acts by binding to the ompC mRNA near the RBS to prevent 30S ribosome loading (19). In Salmonella, MicC downregulates both OmpC and OmpD, binding in the ompD coding sequence to initiate RNase-E dependent mRNA degradation (20). Chen et al. (19)

61 reported that micC transcription is negatively regulated by $O m p R / E n v Z$ in $E$. coli. Transcriptomic 62 data suggested that micC is regulated by OmpR/EnvZ, RpoS, and SlyA in Salmonella (21), but 63 regulation of $m i c C$ has not been characterized in detail. SlyA is a member of MarR/SlyA family of 64 bacterial transcriptional regulators. In Salmonella, slyA mutants are significantly attenuated in the 65 mouse model of infection (22). SlyA acts both positively and negatively to control expression of 
some 30 genes (23-26). SlyA controls some genes independently, but often functions in concert with other transcriptional regulators, including $\operatorname{PhoP}$ and $\operatorname{OmpR}(23,27,28)$.

In this study, we define a new regulatory role for MicC sRNA, controlling the SPI1 T3SS in Salmonella. We found that MicC base pairs with the leader sequence of hilD mRNA to negatively regulate translation of hilD. MicC-mediated SPI1 regulation is dependent on environmental signals and regulated through both SlyA and the OmpR/EnvZ two-component system, which acts through or in conjunction with SlyA. We also show that MicC-dependent regulation of SPI1 is important during intestinal infection.

\section{RESULTS}

75 The small RNA MicC downregulates the expression of HilD. Several regulatory proteins and signals affecting expression of the SPI1 T3SS act at the level of hilD mRNA translation (7). In the few cases that have been characterized, this regulation is mediated either by the RNA binding protein CsrA (29) or sRNAs (30). We previously screened a set of highly conserved sRNAs for those that decrease hilD translation when overproduced, and subsequently characterized regulation of HilD translation by FnrS and ArcZ (8). This screen also identified the 109 nucleotide sRNA MicC, first characterized as a regulator of $o m p C$, encoding the $\mathrm{OmpC}$ porin protein in $E$. coli (19). MicC is encoded in the intergenic region downstream of the pyruvate-flavodoxin oxidoreductase gene (nifJ) in both E. coli and Salmonella and is conserved in the Enterobacteriaceae (Fig. 2A). In Salmonella, MicC downregulates translation of the ompC and ompD mRNAs by base pairing using the highly conserved 5' 20-30 nucleotides (20).

To understand the regulation of the SPI1 T3SS system of Salmonella by MicC, we overexpressed MicC from the pBRplac plasmid (31) in Salmonella strains harboring either an in locus hilD'-'lacZ translational fusion or a hilA'-lacZ' transcriptional fusion. Note that the hilD fusion strain is a hilD null. Thus, this fusion represents the basal level of transcription and is not autoregulated. The Salmonella cultures were inoculated in No Salt LB (NSLB) overnight and subcultured in High Salt LB (HSLB) for 3 hrs to induce SPI1. The expression of hilD was downregulated $\sim 3$-fold in the pMicC strain (Fig. 2B). Expression of hilA was decreased 10 -fold by

93 MicC (Fig. 2B). These data suggest that MicC negatively regulates HilD expression, leading to a

94 more dramatic effect on hilA transcription, consistent with the feed-forward loop model (Fig. 1).

95 To ensure that this is a direct effect on hilD, we introduced the MicC plasmid into an E. coli strain

96 containing a $\mathrm{P}_{\mathrm{BAD}}-h i l D^{\prime}$-'lacZ translational fusion with an arabinose-inducible promoter. The fusion

97 consists of the 35-nt 5' UTR and the first 11 codons of hilD fused in-frame to lacZ. Overexpression 
of MicC in E. coli downregulated the expression of hilD more than 2-fold (Fig. 2B). These results suggest that MicC acts directly on the hilD mRNA to inhibit translation.

100 MicC targets the 5' UTR of hilD mRNA by direct base pairing. Bioinformatic analysis using IntraRNA (http://rna.informatik.uni-freiburg.de/IntaRNA/Input.jsp) suggested that MicC has a binding site in the hilD mRNA immediately upstream of the ribosome binding site (RBS; Fig. 3A). Based on this prediction, we mutated nucleotides 1 to 5 and 11 to 14 as indicated in the pMicC plasmid. We measured $\beta$-galactosidase activity in the Salmonella hilD'-'lacZ translational fusion strain expressing the MicC mutant (pMicC-mt). The pMicC-mt plasmid conferred no significant repression of hilD (Fig. 3B). We then introduced the pMicC-mt plasmid into the E. coli $\mathrm{P}_{\mathrm{BAD}}-h_{i l D}$ regulate hilD expression (Fig. 3C). Based on the predicted interaction, we introduced compensatory mutations in nucleotides -18 to -26 of hilD in the $P_{B A D}-h i l D$ '-'lacZ fusion (Fig. 3A).

110 Overexpression of the wild type MicC had no significant effect on this fusion, whereas the MicC-

111 mt downregulated the mutant hilD mRNA (Fig. 3C). These data support the proposed base-pairing 112 interaction between MicC and the hilD mRNA. It should be noted that several point mutations and

113 double mutations did not affect the interaction, suggesting that the base-pairing is relatively 114 robust. Similar robust base-pairing interactions were also observed between sRNA MicC and 115 ompC mRNA (19) and ompD mRNA (20) targets.

116 The data above show that MicC affects translation of hilD. We also tested the effect of 117 overexpressed MicC on hilC and rtsA using translational LacZ fusions in Salmonella. In hilD 118 strains, expression of MicC led to a decrease in expression of both hilC and rtsA (Fig. 4A). 119 However, there was no effect in the hilD null background, consistent with the fact that MicC inhibits 120 hilD translation (Fig. 4B). Reduced levels of HilD protein decreased transcription of hilC and rtsA, 121 consistent with the feed-forward loop model (Fig. 1). MicC also did not directly affect translation 122 of either hilC or rtsA in E. coli (Fig. 4C). MicC downregulates hilA transcription via HilD (Fig. 2B).

123 To confirm that MicC does not affect hilA translation, we overexpressed MicC in an E. coli strain 124 containing a hilA'-'lacZ translational fusion. MicC had no effect on the expression of this fusion 125 (Fig. 4C). All of these results are consistent with MicC solely regulating hilD translation to affect 126 transcription of hilC, rts $A$ and hilA.

127 MicC requires Hfq but not RNase E for hilD mRNA regulation. The RNA chaperone Hfq is a 128 vital facilitator of sRNA-mRNA imperfect base-pairing (32). To test if the MicC-hilD mRNA 129 interaction is dependent on Hfq, we measured hilD expression levels in an hfq mutant Salmonella 130 after introducing the MicC plasmid. There was no significant regulation mediated by MicC in the 131 hfq background, suggesting that the interaction and perhaps MicC stability require the Hfq 
132 chaperone protein (Fig. 5A). Consistent with our result, Hfq is essential for MicC-dependent

133 regulation OmpC in E. coli (19) and OmpD in Salmonella (20).

134 Negative regulation by sRNAs can be due to simple inhibition of translation initiation or

135 initiation of mRNA degradation by RNaseE. We measured the effects of MicC overproduction on

136 the Salmonella hilD'-'lacZ fusion in an rne131 background strain, which has a defect in RNA

137 degradosome assembly (8, 33-35). Although absolute expression of the hilD fusion was increased

138 in the rne131 background, MicC was still able to regulate (Fig. 5B). Therefore, we conclude that

139 MicC base-pairing to the hilD mRNA blocks translation initiation but does not induce mRNA 140 degradation.

141 MicC expression is activated by SlyA and repressed by OmpR/EnvZ. Studies in E. coli (19)

142 and transcriptomic analysis in Salmonella (21) suggested that MicC expression is repressed by

$143 \mathrm{OmpR} / \mathrm{EnvZ}$ and activated by SlyA. To characterize this regulation in more detail, we examined

144 expression of a micC'-lacZ ${ }^{+}$fusion. Deletion of either envZ or ompR in the fusion strain resulted

145 in increased transcription of MicC in the exponential growth phase (Fig. 6A), confirming that the

146 OmpR/EnvZ two-component system negatively regulates MicC. Deletion of slyA, in contrast,

147 caused a 3-fold decrease in expression, showing that SlyA is a positive regulator of micC

148 expression. Importantly, in the absence of SlyA, deletion of ompR or envZ had no effect,

149 suggesting that OmpR/EnvZ function through, or are at least dependent on, SlyA for their control

150 of micC transcription (Fig. 6A).

151 To determine how this regulation affects SPI1 gene expression, we examined both a hilD'-

152 'lacZ translational (Fig. 6B) and hilA'-lacZ+ transcriptional fusions (Fig. 6C). Deletion of micC

153 caused increased expression of both the hilD and hilA fusions, showing that MicC is affecting hilD

154 translation under these conditions. As expected, deletion of slyA also led to an increase in

155 expression of the hilD and hilA fusions, and deletion of mic $C$ in the slyA background had no further

156 effect. These results show that SlyA is affecting SPI1 expression through MicC-mediated control

157 of hilD translation.

158 Regulation by OmpR/EnvZ is more complicated. Deletion of envZ led to decreased 159 expression of the hilD'-'lacZ fusion, but this decreased expression was also seen in the envZ slyA 160 micC background (Fig 6B). It is interesting to note that deletion of envZ has a greater effect than 161 mutations in ompR, as noted previously $(7,13)$. Note also that our $\Delta o m p R:: C m$ allele is polar on 162 the translationally coupled envZ (36). Thus, this decreased expression of the hilD'-'lacZ fusion 163 seen in the envZ mutant is functioning through OmpR, but is independent of MicC.

164 Deletion of envZ also caused decreased expression of hilA (Fig 6C) that is independent 165 of SlyA and MicC. Loss of OmpR (and EnvZ) has no effect under these conditions. Our previous 
data (7) showed that the primary effect of the envZ mutation in late stationary phase was to decrease HilD protein activity, leading to decreased expression of hilA. In the exponential phase data shown here, we also see an apparent effect on hilD transcription in the envZ mutant; we do not understand the mechanism. Thus OmpR/EnvZ, although controlling micC expression, also affect SPI1 independent of MicC, apparently through several mechanisms, which complicates interpretation of the data.

Transcriptomic data also implicated the sigma factor RpoS in the regulation of micC (21). As shown in Fig. S1A, deletion of rpoS caused increased expression of micC in early stationary phase but has no effect in exponential phase. Given that RpoS is acting negatively to control micC, this is likely an indirect effect. Deletion of rpoS also led to increased hilD transcription, particularly in stationary phase. This regulation was unaffected by loss of MicC (Fig. S1B). Thus, RpoS negatively regulates hilD by an unknown, and likely indirect mechanism (Fig. S1B), but this regulation is independent of $\mathrm{MicC}$.

MicC is negatively regulated by $O m p R / E n v Z$ and negatively regulates $O m p C$ translation. Therefore, it was proposed to play a role in the differential osmoregulation of the porin proteins (19). As such, one would predict that MicC would be preferentially expressed at low osmolarity. We tested this hypothesis by examining expression of the mic $C^{\prime}-l a c Z^{+}$fusion in low and high salt LB (Fig. S2). As shown in Fig. S2B, expression of micC is increased in high salt. Moreover, this regulation is largely independent of OmpR/EnvZ. Thus, overall regulation of micC is inconsistent with a simple role in regulation of the porins in response to osmolarity.

Deletion of MicC enhances SPI1 dependent fitness in vivo. MicC regulates expression of the SPI1 T3SS via direct base pairing with the hilD mRNA. In vitro, this regulation is evident at midto late-exponential phase (Fig. 6). To determine if MicC affects SPI1 regulation in vivo in a manner that affects virulence, we performed competition assays using BALB/C mice. In oral infections, the $\Delta$ micC strain outcompeted the wildtype strain in both the upper small intestine (includes duodenum and jejunum) and lower small intestine (includes ileum) (Fig. 7A), the primary site of Salmonella invasion into epithelium cells $(2,3,37)$. There was no significant fitness advantage for bacteria recovered from the spleen after either oral or intraperitoneal (IP) infection (Fig. 7B); systemic infection does not require SPI1 $(5,38)$. To determine whether the observed effects in the intestine were due to changes in SPI1 expression, we also performed competition assays in strains lacking the SPI1 T3SS. In the $\triangle$ spi1 background, deletion of mic $C$ had no significant effect in the competition assay (Fig. 7C). These data are consistent with MicC having a significant regulatory role on hilD translation during intestinal infection. 


\section{DISCUSSION}

The SPI1 T3SS is regulated in response to a plethora of physiological and environmental factors to ensure that this critical virulence factor is expressed at the appropriate time and place in the host and to optimize that expression. In this study, we identified the sRNA, MicC, as a repressor of hilD translation. MicC was previously identified as a negative regulator of the outer membrane porin proteins $\mathrm{OmpC}(19)$ and $\mathrm{OmpD}(20)$. MicC acts in the canonical fashion to control hilD translation, base pairing just upstream of the ribosome binding site (Fig. 3A). This binding blocks translation per se rather than initiating mRNA degradation (Fig. 3B). MicC also base pairs just upstream of the ribosome binding site in the ompC mRNA to block translation (19). Interestingly, in the case of $\mathrm{mpD}$, MicC base pairs starting at 67 nucleotides downstream of the AUG and acts by initiating mRNA degradation versus blocking translation (20). MicC does not directly regulate hilC, rtsA or hilA (Fig. 4), showing that MicC-mediated downregulation of SPI1 T3SS is solely through regulation of hilD mRNA translation. These data reinforce HilD as the primary site of signal integration in the SPI1 regulatory circuit (Fig. 1).

Expression data in E. coli and transcriptome data in Salmonella suggested that micC transcription is controlled by SlyA, OmpR/EnvZ, and RpoS (21). Our data suggest that the primary transcriptional activator of micC is SlyA (Fig. 6A). OmpR/EnvZ negatively regulate micC transcription by affecting SlyA activation. Whether this regulation is all occurring directly at the micC promoter will require further investigation, but SlyA often works in conjunction with other transcriptional regulators in the control of gene expression $(23,24,27,28)$. Comparison of the sequence upstream of micC in various Enterobacteriaceae reveals a strikingly conserved sequence between -31 and -50 from the transcription start site (Fig. 2A). This suggests that this sequence is important for regulation, but it matches neither the SlyA (39-41) nor the OmpR consensus sequence (42). We also show that RpoS negatively regulates micC transcription in early stationary phase. This is almost certainly an indirect effect and determining the mechanism will also require further analyses.

The physiological signals that influence SlyA are unclear, although salicylate binds to and

$227(25,43,44)$. SlyA is strongly induced when Salmonella is replicating in macrophages and slyA 228 mutants are not able to survive in macrophages and are, therefore, attenuated for virulence (28). 229 Our data show that SlyA increases the expression of MicC, which helps to repress the SPI1 T3SS.

230 This regulation is apparently evident in the intestine with the micC mutant outcompeting the 231 wildtype, consistent with increased expression of the SPI1 T3SS. The SPI1 T3SS is neither 232 expressed nor required during systemic infection and replication in macrophages $(5,45)$. Our data 
233 suggest that this strong negative regulation of the system is mediated primarily by PhoPQ (46),

234 but activation of MicC by SlyA may contribute to the downregulation of hilD transcription in the

235 intracellular environment.

236 MicC, which is negatively regulated by $\mathrm{OmpR}$ and blocks ompC translation, was proposed

237 to facilitate regulation of $\mathrm{OmpF}$ and $\mathrm{OmpC}$ in response to osmolarity (19). OmpR/EnvZ

238 differentially regulate transcription of the porin genes such that $o m p F$ is preferentially transcribed

239 in low osmolarity and $\mathrm{ompC}$ is preferentially transcribed in high osmolarity $(12,47)$. The sRNA

$240 \mathrm{MicF}$ is co-transcribed with ompC and blocks ompF translation (18). Logic would dictate that MicC

241 should be produced preferentially in low osmolarity to down regulate OmpC expression under

242 these conditions. Interestingly, our results show that mic $C$ transcription increases with osmolarity

243 (at least under the conditions we examined; Fig. S2) and that this regulation is independent of

244 OmpR. Thus, the simple model does not hold. Indeed, OmpR is now known to be a global

245 transcriptional regulator and most genes in the OmpR regulon are not osmoregulated (48), but

246 rather activated at some threshold level of OmpR-P. Only if that threshold level is high, as in the

247 case of $o m p C$, is the gene preferentially activated at high osmolarity. Transcriptional regulation of

$248 \mathrm{ompF}$ is more complex and apparently unique, being activated at low levels of OmpR-P, but then

249 actively repressed by OmpR-P at higher levels (47-49). Negative regulation of micC by

250 OmpR/EnvZ is via SlyA and the overall osmoregulation of micC is independent of the two-

251 component system.

252 OmpR/EnvZ regulation of SPI1 is more complicated and one can argue that low levels of 253 OmpR-P play a role. Deletion of EnvZ leads to a significant decrease in hilA transcription (Fig. 6).

254 This effect functions through OmpR; the ompR mutation is polar on envZ (36). It has long been

255 known that loss of EnvZ, but not OmpR/EnvZ, affects SPI1 expression $(5,7,14)$. We previously

256 showed that this envZ phenotype is mediated through control of HilD protein activity (7), which is

257 consistent with the data shown here. However, those previous experiments were performed in

258 late stationary phase. In the exponential phase experiments shown here, we also see an effect

259 on hilD expression in the hilD null strain. In E. coli, there are low levels of OmpR-P in the envZ

260 null strain, due to phosphorylation of OmpR by acetyl phosphate (50-52). Thus, it appears that

261 low levels of OmpR-P are actively leading to decreased HilD protein activity (7) and perhaps hilD

262 transcription/translation through unknown mechanisms. This is consistent with overall activation

263 of SPI1 in high osmolarity, which would further be enhanced by OmpR-mediated repression of

264 micC.

265 These results emphasize the role of HilD as the major signal integration point for control 266 of the SPI1 T3SS. Most regulatory input is via post-transcriptional control of HilD, affecting HilD 
267 activity via protein-protein interactions, hilD translation, or mRNA stability $(6-8,29,53)$, although

268 the mechanisms are understood in only a few cases. We know that hilD translation is affected by

269 binding of the RNA binding protein CsrA in the 39 nt hilD 5' UTR $(29,54)$. Translation initiation is

270 also controlled by the sRNAs FnrS, ArcZ (8) and MicC, all of which base pair at the ribosome

271 binding site. All three of these sRNAs affect SPI1 expression during intestinal infection in the

272 animal. More detailed analyses are required to determine the mechanisms and physiological role

273 of additional sRNAs identified as affecting overall control of the T3SS (8).

\section{MATERIAL AND METHODS}

275 Bacterial strains and growth conditions. Bacterial strains and plasmids used in this study are

276 described in Table S1. All Salmonella enterica serovar Typhimurium strains are isogenic 277 derivatives of strain 14028 [American Type Culture Collection (ATCC)] and were constructed 278 using P22 HT105/1 int-201 (P22)- mediated transduction. Deletion of genes or insertion of 279 antibiotic resistance cassettes was performed using $\lambda$-red mediated recombination $(55,56)$. 280 Insertions and deletions were confirmed by PCR and mutations were transferred into unmutagenized backgrounds by P22 transduction. In some cases, antibiotic resistance cassettes were removed using the temperature-sensitive plasmid pCP20 carrying the FLP recombinase (57). To create transcriptional lacZ fusions to MicC, the insertion mutation in MicC was converted to a transcriptional lac fusion using FLP-mediated recombination with plasmid pKG136, as previously described (56). The translational lacZ reporter fusions in $E$. coli were constructed using lambda Red-mediated recombination in strain PM1205, as described previously $(8,31)$.

Strains were routinely cultured in lysogeny broth (LB; $10 \%$ tryptone, $5 \%$ yeast extract, $5 \%$ $\mathrm{NaCl}$ ). For SPI1 expression experiments, cells were grown in No Salt LB (NSLB; $10 \%$ tryptone, $5 \%$ yeast extract, $0 \% \mathrm{NaCl}$ ) or High Salt LB (HSLB; $10 \%$ tryptone, $5 \%$ yeast extract, $10 \% \mathrm{NaCl}$ ). All strains were grown at $37^{\circ} \mathrm{C}$ with aeration, except for the strains containing the temperature-

292 following final concentrations: ampicillin (Ap, $100 \mu \mathrm{g} / \mathrm{mL})$, kanamycin $(\mathrm{Km}, 50 \mu \mathrm{g} / \mathrm{mL})$, 293 chloramphenicol $(\mathrm{Cm}, 20 \mu \mathrm{g} / \mathrm{mL})$, apramycin (Apr, $50 \mu \mathrm{g} / \mathrm{mL}$ ) and tetracycline (Tet, $10 \mu \mathrm{g} / \mathrm{mL})$.

294 Construction of plasmids and site-directed mutagenesis. The MicC small RNA was amplified 295 from the S. Typhimurium 14028 genome using oligonucleotides pairs F-Aatll-MicC and R-EcoRI296 MicC and cloned into the pBR-plac vector (31) after digestion with Aatll and EcoRI restriction 297 enzymes, creating pMicC. The hilD mRNA/MicC sRNA interactions were predicted using the 298 IntaRNA RNA-RNA interaction tool (58). Mutations were introduced into the pMicC plasmid using 
299 a QuikChange Lightning site-directed mutagenesis kit (Stratagene). Oligonucleotides used in this 300 study are listed in Table S2.

301 ß-Galactosidase assays. ß-Galactosidase assays were performed using a microtiter plate assay

302 as previously described (49). Briefly, Salmonella strains were inoculated in NSLB medium and 303 grown $\mathrm{ON}$ at $37^{\circ} \mathrm{C}$ on a roller drum. These cultures were subsequently diluted 1:100 into $2 \mathrm{ml}$ of 304 HSLB medium and grown at $37^{\circ} \mathrm{C}$ on a roller drum for $3 \mathrm{hr}$ or $8 \mathrm{hr}$ (where indicated). For E. coli 305 cultures, strains were initially inoculated into LB and grown overnight, then subcultured 1:100 into $3062 \mathrm{ml}$ of LB medium with 100 MM IPTG and $0.001 \%$ arabinose and grown at $37^{\circ} \mathrm{C}$ on a roller drum 307 for $3 \mathrm{hr}$. ß-Galactosidase activity units are defined as [ $\mu \mathrm{mol}$ of orthonitrophenol (ONP) formed $\left.308 \mathrm{~min}^{-1}\right] \times 10 \% /\left(\mathrm{OD}_{600} \times \mathrm{ml}\right.$ of cell suspension).

309 In vitro and in vivo competition assays. All animal work was reviewed and approved by the 310 University of Illinois Institutional Animal Care and Use Committee (IACUC). Procedures were 311 performed in our AAALAC accredited facility in accordance with University and PHS guidelines 312 under protocol 15214. Competition assays in vivo and in vitro were performed using isogenic wild 313 type and $\Delta$ micC, or $\Delta$ SPI1 and $\Delta$ micC $\Delta$ SPI1 mutants. Briefly, strains were grown overnight in LB. 314 For oral infections, strains were mixed 1:1, washed, and suspended in $0.1 \mathrm{M}$ phosphate buffered 315 saline $(\mathrm{pH} 8)$ to an adjusted cfu $\mathrm{ml}^{-1}$ of $5 \times 10^{8}$ (for wild type vs $\Delta$ micC) or $10^{9}$ (for $\Delta$ SPI1 vs $\Delta$ micC $316 \Delta$ SPI1). Before infection, food and water were withheld for $4 \mathrm{~h}$, and then mice were inoculated 317 with $200 \mu$ l of cell suspension by oral gavage. For intraperitoneal infections, 1:1 cell mixtures were 318 diluted to $10^{3} \mathrm{cfu} \mathrm{ml}^{-1}$ in phosphate buffered saline $(\mathrm{pH} 7)$. Mice were inoculated with $200 \mu \mathrm{l}$ of 319 cell suspension by intraperitoneal (IP) injection. All inocula were diluted and plated on LB and 320 then replica plated to appropriate antibiotic medium to determine the exact ratios of strains. After 3213.5 days of infection, mice were sacrificed by $\mathrm{CO}_{2}$ asphyxiation and the spleens and small 322 intestines were dissected from orally infected mice, or the spleens were dissected from IP infected 323 mice. Tissues were mechanically homogenized in PBS with 15\% glycerol and appropriate 324 dilutions were plated on LB containing the appropriate antibiotics and subsequently replica plated 325 to determine the ratio of strains recovered. In vitro competitions were performed simultaneously 326 by subculturing $10^{3} \mathrm{cfu}$ of the same inocula used for the in vivo experiments into $2 \mathrm{~mL}$ of LB. The 327 cultures were incubated overnight at $37{ }^{\circ} \mathrm{C}$ with aeration, diluted, and plated on LB. Resulting 328 colonies were replica plated onto LB containing the appropriate antibiotics. Results are presented 329 as competitive index $(\mathrm{Cl})$, calculated as (percentage of strain A recovered/percentage of strain $B$ 330 recovered)/(percentage of strain A inoculated/percentage of strain B inoculated).

\section{ACKNOWLEDGEMENTS}

332 This work was funded by NIH grant R01 GM120182 to JMS and CKV. 


\section{REFERENCES}

1. G. B. D. Non-Typhoidal Salmonella Invasive Disease Collaborators. 2019. The global burden of non-typhoidal salmonella invasive disease: a systematic analysis for the Global Burden of Disease Study 2017. Lancet Infect Dis 19:1312-1324. https://doi.org/10.1016/S1473-3099(19)30418-9.

2. Carter PB, Collins FM. 1974. The route of enteric infection in normal mice. J Exp Med 139:1189-203. https://doi.org/10.1084/jem.139.5.1189.

3. Clark MA, Jepson MA, Simmons NL, Hirst BH. 1994. Preferential interaction of Salmonella typhimurium with mouse Peyer's patch M cells. Res Microbiol 145:543-52.

https://doi.org/10.1016/0923-2508(94)90031-0.

4. Galan JE, Lara-Tejero M, Marlovits TC, Wagner S. 2014. Bacterial type III secretion systems: specialized nanomachines for protein delivery into target cells. Annu Rev Microbiol 68:415-38. https://doi.org/10.1146/annurev-micro-092412-155725.

5. Ellermeier CD, Ellermeier JR, Slauch JM. 2005. HilD, HilC and RtsA constitute a feed forward loop that controls expression of the SPI1 type three secretion system regulator hilA in Salmonella enterica serovar Typhimurium. Mol Microbiol 57:691-705. https://doi.org/10.1111/j.1365-2958.2005.04737.x.

6. Grenz JR, Cott Chubiz JE, Thaprawat P, Slauch JM. 2018. HilE Regulates HilD by Blocking DNA Binding in Salmonella enterica Serovar Typhimurium. J Bacteriol 200. https://doi.org/10.1128/JB.00750-17.

7. Golubeva YA, Sadik AY, Ellermeier JR, Slauch JM. 2012. Integrating global regulatory input into the Salmonella pathogenicity island 1 type III secretion system. Genetics 190:79-90. https://doi.org/10.1534/genetics.111.132779.

8. Kim K, Golubeva YA, Vanderpool CK, Slauch JM. 2019. Oxygen-dependent regulation of SPI1 type three secretion system by small RNAs in Salmonella enterica serovar Typhimurium. Mol Microbiol 111:570-587. https://doi.org/10.1111/mmi.14174.

9. Hor J, Matera G, Vogel J, Gottesman S, Storz G. 2020. Trans-Acting Small RNAs and Their Effects on Gene Expression in Escherichia coli and Salmonella enterica. EcoSal Plus 9. https://doi.org/10.1128/ecosalplus.ESP-0030-2019.

10. Moller T, Franch T, Hojrup P, Keene DR, Bachinger HP, Brennan RG, Valentin-Hansen P. 2002. Hfq: a bacterial Sm-like protein that mediates RNA-RNA interaction. Mol Cell 9:23-30. https://doi.org/10.1016/s1097-2765(01)00436-1. 
11. De Lay N, Schu DJ, Gottesman S. 2013. Bacterial small RNA-based negative regulation: Hfq and its accomplices. J Biol Chem 288:7996-8003. https://doi.org/10.1074/jbc.R112.441386.

12. Slauch JM, Silhavy TJ. 1997. The porin regulon: a paradigm for the two-component regulatory systems, p 383-417. In Lin ECC, Lynch AS (ed), Regulation of gene expression in Escherichia coli doi:10.1007/978-1-4684-8601-8_19. R.G. Landes Co., Austin, TX.

13. Lee AK, Detweiler CS, Falkow S. 2000. OmpR regulates the two-component system SsrAssrB in Salmonella pathogenicity island 2. J Bacteriol 182:771-81. https://doi.org/10.1128/JB.182.3.771-781.2000.

14. Lucas RL, Lee CA. 2001. Roles of hilC and hilD in regulation of hilA expression in Salmonella enterica serovar Typhimurium. J Bacteriol 183:2733-45. https://doi.org/10.1128/JB.183.9.2733-2745.2001.

15. Feng X, Oropeza R, Kenney LJ. 2003. Dual regulation by phospho-OmpR of ssrA/B gene expression in Salmonella pathogenicity island 2. Mol Microbiol 48:1131-43. https://doi.org/10.1046/.1365-2958.2003.03502.x.

16. Mattison K, Kenney LJ. 2002. Phosphorylation alters the interaction of the response regulator OmpR with its sensor kinase EnvZ. J Biol Chem 277:11143-8. https://doi.org/10.1074/jbc.M111128200.

17. Andersen J, Delihas N, Ikenaka K, Green PJ, Pines O, Ilercil O, Inouye M. 1987. The isolation and characterization of RNA coded by the micF gene in Escherichia coli. Nucleic Acids Res 15:2089-101. https://doi.org/10.1093/nar/15.5.2089.

18. Aiba H, Matsuyama S, Mizuno T, Mizushima S. 1987. Function of micF as an antisense RNA in osmoregulatory expression of the ompF gene in Escherichia coli. J Bacteriol 169:3007-12. https://doi.org/10.1128/jb.169.7.3007-3012.1987.

19. Chen S, Zhang A, Blyn LB, Storz G. 2004. MicC, a second small-RNA regulator of Omp protein expression in Escherichia coli. J Bacteriol 186:6689-97. https://doi.org/10.1128/JB.186.20.6689-6697.2004.

20. Pfeiffer V, Papenfort K, Lucchini S, Hinton JC, Vogel J. 2009. Coding sequence targeting by MicC RNA reveals bacterial mRNA silencing downstream of translational initiation. Nat Struct Mol Biol 16:840-6. https://doi.org/10.1038/nsmb.1631.

21. Colgan AM, Kroger C, Diard M, Hardt WD, Puente JL, Sivasankaran SK, Hokamp K, Hinton JC. 2016. The Impact of 18 Ancestral and Horizontally-Acquired Regulatory Proteins upon the Transcriptome and sRNA Landscape of Salmonella enterica serovar Typhimurium. PLoS Genet 12:e1006258. https://doi.org/10.1371/journal.pgen.1006258. 
22. Libby SJ, Goebel W, Ludwig A, Buchmeier N, Bowe F, Fang FC, Guiney DG, Songer JG, Heffron F. 1994. A cytolysin encoded by Salmonella is required for survival within macrophages. Proc Natl Acad Sci U S A 91:489-93. https://doi.org/10.1073/pnas.91.2.489.

23. Navarre WW, Halsey TA, Walthers D, Frye J, McClelland M, Potter JL, Kenney LJ, Gunn JS, Fang FC, Libby SJ. 2005. Co-regulation of Salmonella enterica genes required for virulence and resistance to antimicrobial peptides by SlyA and PhoP/PhoQ. Mol Microbiol 56:492-508. https://doi.org/10.1111/j.1365-2958.2005.04553.x.

24. Spory A, Bosserhoff A, von Rhein C, Goebel W, Ludwig A. 2002. Differential regulation of multiple proteins of Escherichia coli and Salmonella enterica serovar Typhimurium by the transcriptional regulator SlyA. J Bacteriol 184:3549-59. https://doi.org/10.1128/JB.184.13.3549-3559.2002.

25. Will WR, Brzovic P, Le Trong I, Stenkamp RE, Lawrenz MB, Karlinsey JE, Navarre WW, Main-Hester K, Miller VL, Libby SJ, Fang FC. 2019. The Evolution of SlyA/RovA Transcription Factors from Repressors to Countersilencers in Enterobacteriaceae. mBio 10. https://doi.org/10.1128/mBio.00009-19.

26. Ellison DW, Miller VL. 2006. Regulation of virulence by members of the MarR/SlyA family. Curr Opin Microbiol 9:153-9. https://doi.org/10.1016/j.mib.2006.02.003.

27. Shi Y, Latifi T, Cromie MJ, Groisman EA. 2004. Transcriptional control of the antimicrobial peptide resistance ugtL gene by the Salmonella PhoP and SlyA regulatory proteins. J Biol Chem 279:38618-25. https://doi.org/10.1074/jbc.M406149200.

28. Linehan SA, Rytkonen A, Yu XJ, Liu M, Holden DW. 2005. SlyA regulates function of Salmonella pathogenicity island 2 (SPI-2) and expression of SPI-2-associated genes. Infect Immun 73:4354-62. https://doi.org/10.1128/IAI.73.7.4354-4362.2005.

29. Martinez LC, Yakhnin H, Camacho MI, Georgellis D, Babitzke P, Puente JL, Bustamante VH. 2011. Integration of a complex regulatory cascade involving the SirA/BarA and Csr global regulatory systems that controls expression of the Salmonella SPI-1 and SPI-2 virulence regulons through HilD. Mol Microbiol 80:1637-56. https://doi.org/10.1111/j.13652958.2011.07674.x.

30. Kim K, Palmer AD, Vanderpool CK, Slauch JM. 2019. The Small RNA PinT Contributes to PhoP-Mediated Regulation of the Salmonella Pathogenicity Island 1 Type III Secretion System in Salmonella enterica Serovar Typhimurium. J Bacteriol 201. https://doi.org/10.1128/JB.00312-19. 
31. Mandin P, Gottesman S. 2009. A genetic approach for finding small RNAs regulators of genes of interest identifies RybC as regulating the DpiA/DpiB two-component system. Mol Microbiol 72:551-65. https://doi.org/10.1111/j.1365-2958.2009.06665.x.

32. Vogel J, Luisi BF. 2011. Hfq and its constellation of RNA. Nat Rev Microbiol 9:578-89. https://doi.org/10.1038/nrmicro2615.

33. Vanzo NF, Li YS, Py B, Blum E, Higgins CF, Raynal LC, Krisch HM, Carpousis AJ. 1998. Ribonuclease $E$ organizes the protein interactions in the Escherichia coli RNA degradosome. Genes Dev 12:2770-81. https://doi.org/10.1101/gad.12.17.2770.

34. Lopez PJ, Marchand I, Joyce SA, Dreyfus M. 1999. The C-terminal half of RNase E, which organizes the Escherichia coli degradosome, participates in mRNA degradation but not rRNA processing in vivo. Mol Microbiol 33:188-99. https://doi.org/10.1046/j.13652958.1999.01465.x.

35. Viegas SC, Pfeiffer V, Sittka A, Silva IJ, Vogel J, Arraiano CM. 2007. Characterization of the role of ribonucleases in Salmonella small RNA decay. Nucleic Acids Res 35:7651-64. https://doi.org/10.1093/nar/gkm916.

36. Liljestrom P, Laamanen I, Palva ET. 1988. Structure and expression of the ompB operon, the regulatory locus for the outer membrane porin regulon in Salmonella typhimurium LT-2. J Mol Biol 201:663-73. https://doi.org/10.1016/0022-2836(88)90465-2.

37. Jones BD, Ghori N, Falkow S. 1994. Salmonella typhimurium initiates murine infection by penetrating and destroying the specialized epithelial M cells of the Peyer's patches. J Exp Med 180:15-23. https://doi.org/10.1084/jem.180.1.15.

38. Murray RA, Lee CA. 2000. Invasion genes are not required for Salmonella enterica serovar typhimurium to breach the intestinal epithelium: evidence that salmonella pathogenicity island 1 has alternative functions during infection. Infect Immun 68:5050-5. https://doi.org/10.1128/IAI.68.9.5050-5055.2000.

39. Ballesteros MF, Torrez Lamberti MF, Farizano JV, Pescaretti MM, Delgado MA. 2019. Regulatory Effect of SlyA on rcsB Expression in Salmonella enterica Serovar Typhimurium. J Bacteriol 201. https://doi.org/10.1128/JB.00673-18.

40. Haider F, Lithgow JK, Stapleton MR, Norte VA, Roberts RE, Green J. 2008. DNA recognition by the Salmonella enterica serovar Typhimurium transcription factor SlyA. Int Microbiol 11:245-50. https://doi.org/10.2436/20.1501.01.68.

41. Stapleton MR, Norte VA, Read RC, Green J. 2002. Interaction of the Salmonella typhimurium transcription and virulence factor SlyA with target DNA and identification of 
members of the SlyA regulon. J Biol Chem 277:17630-7.

https://doi.org/10.1074/jbc.M110178200.

42. Huang KJ, Igo MM. 1996. Identification of the bases in the ompF regulatory region, which interact with the transcription factor OmpR. J Mol Biol 262:615-28. https://doi.org/10.1006/jmbi.1996.0540.

43. Dolan KT, Duguid EM, He C. 2011. Crystal structures of SlyA protein, a master virulence regulator of Salmonella, in free and DNA-bound states. J Biol Chem 286:22178-85. https://doi.org/10.1074/jbc.M111.245258.

44. Cabezas CE, Briones AC, Aguirre C, Pardo-Este C, Castro-Severyn J, Salinas CR, Baquedano MS, Hidalgo AA, Fuentes JA, Morales EH, Meneses CA, Castro-Nallar E, Saavedra CP. 2018. The transcription factor SlyA from Salmonella Typhimurium regulates genes in response to hydrogen peroxide and sodium hypochlorite. Res Microbiol 169:263278. https://doi.org/10.1016/j.resmic.2018.04.003.

45. Srikumar S, Kroger C, Hebrard M, Colgan A, Owen SV, Sivasankaran SK, Cameron AD, Hokamp K, Hinton JC. 2015. RNA-seq Brings New Insights to the Intra-Macrophage Transcriptome of Salmonella Typhimurium. PLoS Pathog 11:e1005262. https://doi.org/10.1371/journal.ppat.1005262.

46. Palmer AD, Kim K, Slauch JM. 2019. PhoP-Mediated Repression of the SPI1 Type 3 Secretion System in Salmonella enterica Serovar Typhimurium. J Bacteriol 201. https://doi.org/10.1128/JB.00264-19.

47. Slauch JM, Silhavy TJ. 1989. Genetic analysis of the switch that controls porin gene expression in Escherichia coli K-12. J Mol Biol 210:281-92. https://doi.org/10.1016/00222836(89)90330-6.

48. Kenney LJ, Anand GS. 2020. EnvZ/OmpR Two-Component Signaling: An Archetype System That Can Function Noncanonically. EcoSal Plus 9. https://doi.org/10.1128/ecosalplus.ESP-0001-2019.

49. Slauch JM, Silhavy TJ. 1991. cis-acting ompF mutations that result in OmpR-dependent constitutive expression. J Bacteriol 173:4039-48. https://doi.org/10.1128/jb.173.13.40394048.1991.

50. Bang IS, Kim BH, Foster JW, Park YK. 2000. OmpR regulates the stationary-phase acid tolerance response of Salmonella enterica serovar typhimurium. J Bacteriol 182:2245-52. https://doi.org/10.1128/JB.182.8.2245-2252.2000. 
51. Russo FD, Silhavy TJ. 1991. EnvZ controls the concentration of phosphorylated OmpR to mediate osmoregulation of the porin genes. J Mol Biol 222:567-80. https://doi.org/10.1016/0022-2836(91)90497-t.

52. Slauch JM, Garrett S, Jackson DE, Silhavy TJ. 1988. EnvZ functions through OmpR to control porin gene expression in Escherichia coli K-12. J Bacteriol 170:439-41. https://doi.org/10.1128/jb.170.1.439-441.1988.

53. Chubiz JE, Golubeva YA, Lin D, Miller LD, Slauch JM. 2010. FliZ regulates expression of the Salmonella pathogenicity island 1 invasion locus by controlling HilD protein activity in Salmonella enterica serovar typhimurium. J Bacteriol 192:6261-70. https://doi.org/10.1128/JB.00635-10.

54. Altier C, Suyemoto M, Lawhon SD. 2000. Regulation of Salmonella enterica serovar typhimurium invasion genes by csrA. Infect Immun 68:6790-7. https://doi.org/10.1128/IAI.68.12.6790-6797.2000.

55. Datsenko KA, Wanner BL. 2000. One-step inactivation of chromosomal genes in Escherichia coli K-12 using PCR products. Proc Natl Acad Sci U S A 97:6640-5. https://doi.org/10.1073/pnas.120163297.

56. Ellermeier CD, Janakiraman A, Slauch JM. 2002. Construction of targeted single copy lac fusions using lambda Red and FLP-mediated site-specific recombination in bacteria. Gene 290:153-61. https://doi.org/10.1016/s0378-1119(02)00551-6.

57. Cherepanov PP, Wackernagel W. 1995. Gene disruption in Escherichia coli: TcR and KmR cassettes with the option of Flp-catalyzed excision of the antibiotic-resistance determinant. Gene 158:9-14. https://doi.org/10.1016/0378-1119(95)00193-a.

58. Mann M, Wright PR, Backofen R. 2017. IntaRNA 2.0: enhanced and customizable prediction of RNA-RNA interactions. Nucleic Acids Res 45:W435-W439. https://doi.org/10.1093/nar/gkx279. 


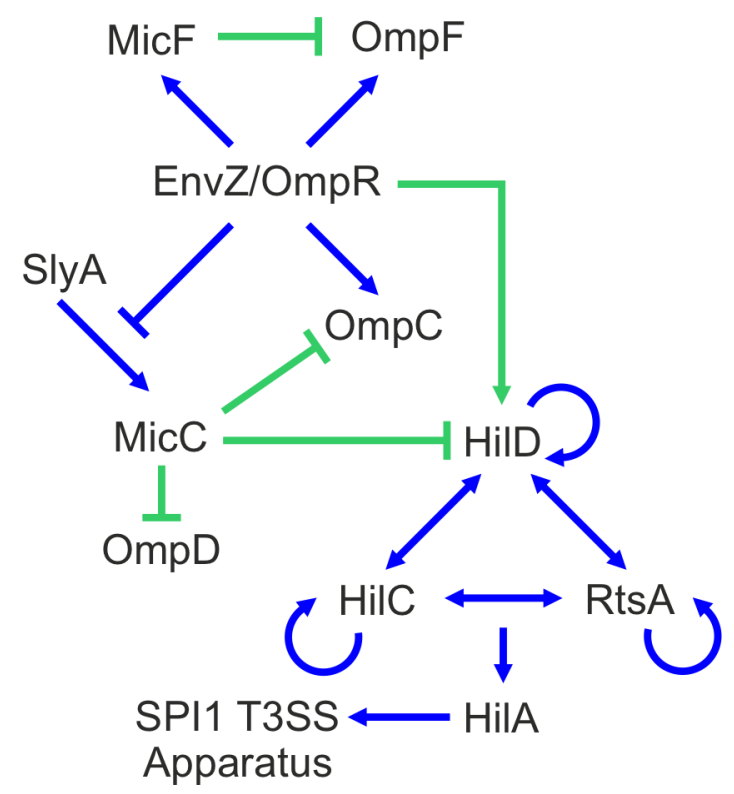

FIG 1. Simplified model of SPI1 T3SS regulatory circuit. Arrows indicate positive regulation, blunt ends indicate negative regulation, blue lines indicate transcriptional regulation, and green lines indicate post-transcriptional regulation. 
bioRxiv preprint doi: https://doi.org/10.1101/2021.08.03.454913; this version posted August 3, 2021. The copyright holder for this preprint (which was not certified by peer review) is the author/funder, who has granted bioRxiv a license to display the preprint in perpetuity. It is made available under aCC-BY-NC-ND 4.0 International license.

A

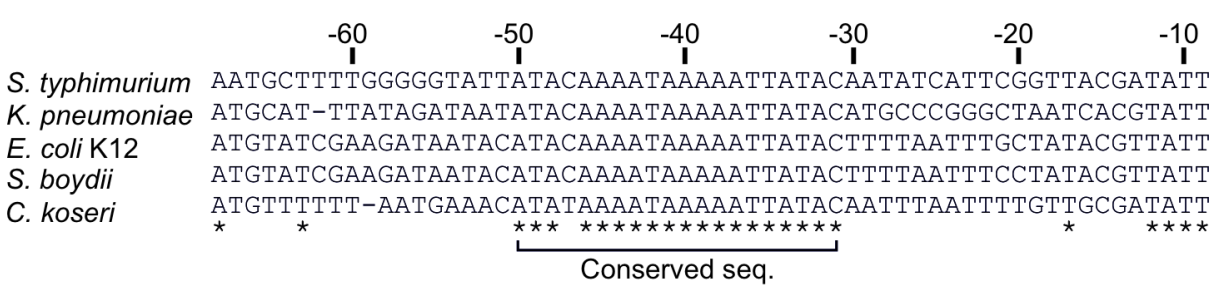

$\begin{array}{cccccc}1 & 10 & 20 & 30 & 40 & 50 \\ \text { S. typhimurium } & \mathbf{I} & \mathbf{I} & \mathbf{I} & \mathbf{I} & \mathbf{I}\end{array}$

K. pneumoniae ATTTGCGCGTTATATGCCTTTATTGTCATGCCAATAATTTATTGTTGCCGTCTCATTCTG

E. coli K12 CTGCGCGGGTTATATGCCTTTATTGTCACAGATTTTATTTTCTGTTGGGCCATTGCATTG

S. boydii CTGCGCGGGTTATATGCCTTTATTGTCACAGATTTTATTTTCTGTTGGGCCATTGCATTG

C. koseri C: ompC

hilD

$m p D$

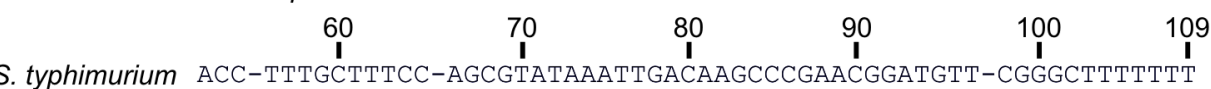

. pneumoniae CGGAATGATGTTGTTTATCGgTAAAACGACAAGCCCGAACGTTGTGTTCGGGCTTTTTT

E. coli K12 -CCACTGATTTTCC-AACATATAAAAAGACAAGCCCGAACAGTCGTC-CGGGCTTTTTTT

S. boydii -CCACTGATTTTCC-AACATATAAAAAGACAAGCCCGAACAGTCGTC-CGGGCTTTTTTT

C. koseri AGTACTGATCTTCC-AGCGAATGAATTGACAAGCCCGAACCAAGGTT-CGGGCTTTTTTT

B

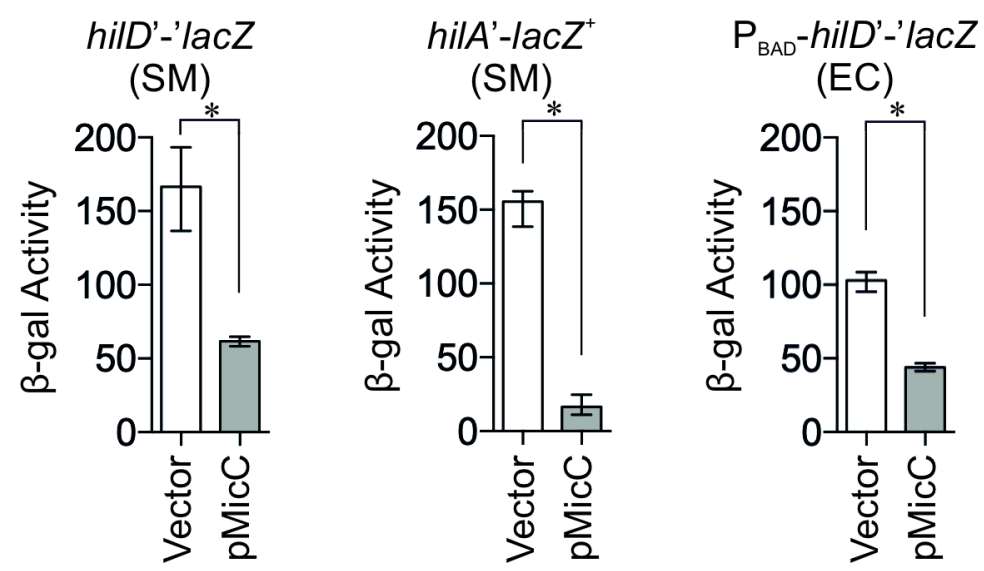

FIG 2. The conserved small RNA MicC negatively regulates the SPI1 T3SS by repressing hilD translation in Salmonella. (A) Alignment of the MicC sequences from various Enterobacteriaceae. The asterisks indicate sequence identity. Sequences corresponding to the regions of MicC that base pair to $o m p C$, ompD and hilD are underlined. (B) $\beta$-gal activity in Salmonella (SM) strains harboring a hilD'-'lacZ translational fusion or a hilA'-lacZ transcriptional fusion, or an E. coli (EC) strain harboring a hilD'-'lacZ translational fusion under control of an arabinose-inducible promoter. Each background contains the pBR-plac vector or pMicC plasmid. $\beta$-gal activity units are defined as ( $\mu \mathrm{mol}$ of ONP formed $\left.\mathrm{min}^{-1}\right) \times 10^{6} /\left(\mathrm{OD}_{600} \times \mathrm{ml}\right.$ of cell suspension). Results are shown as median with interquartile range and asterisks indicate significant differences between the datasets ( $n \geq 4, P<0.05$, using a Mann-Whitney test). Strains used: JS749, JS892 and JMS6500, with plasmids pBR-plac vector or pMicC. 
A

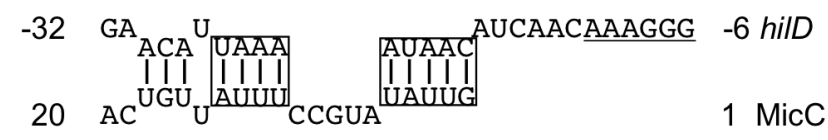

B
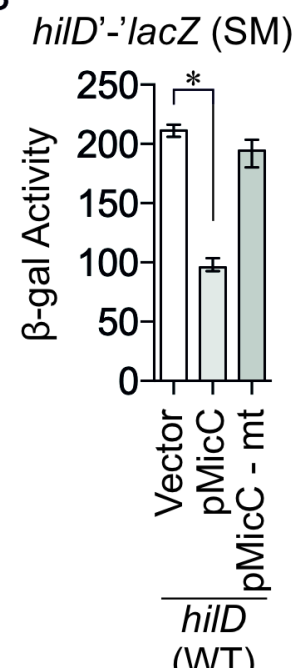

C

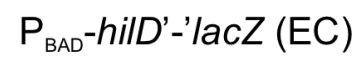
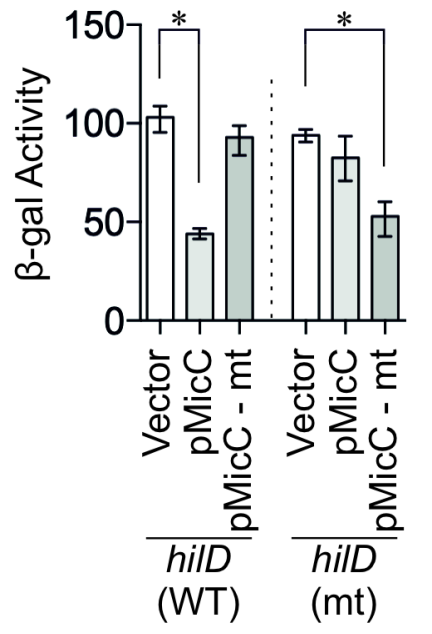

FIG 3. MicC negatively regulates hilD translation by base pairing near the RBS of the hilD mRNA. (A) Predicted base pairing interaction between MicC and hilD mRNA. The RBS is underlined; boxes represent the nucleotides changed in the complementary mutations. (B) $\beta$-gal activity in the Salmonella (SM) strain harboring the wild type hilD'-'lacZ translational fusion with vector pBRplac, wild type pMicC, or mutated pMicC-mt plasmid. (C) $\beta$-gal activity in $E$. coli $(E C)$ strains harboring either the wild type or mutated hilD'-'lacZ translational fusion with empty vector, wild type pMicC, or mutated pMicC-mt plasmid. Results are shown as median with interquartile range. Asterisks indicate significant differences between the datasets ( $n \geq 4, P<0.05$, using a Mann-Whitney test). Strains used: JS892, JMS6500 and JMS6510, with plasmids pBR-plac vector, pMicC, or pMicC-mt. 

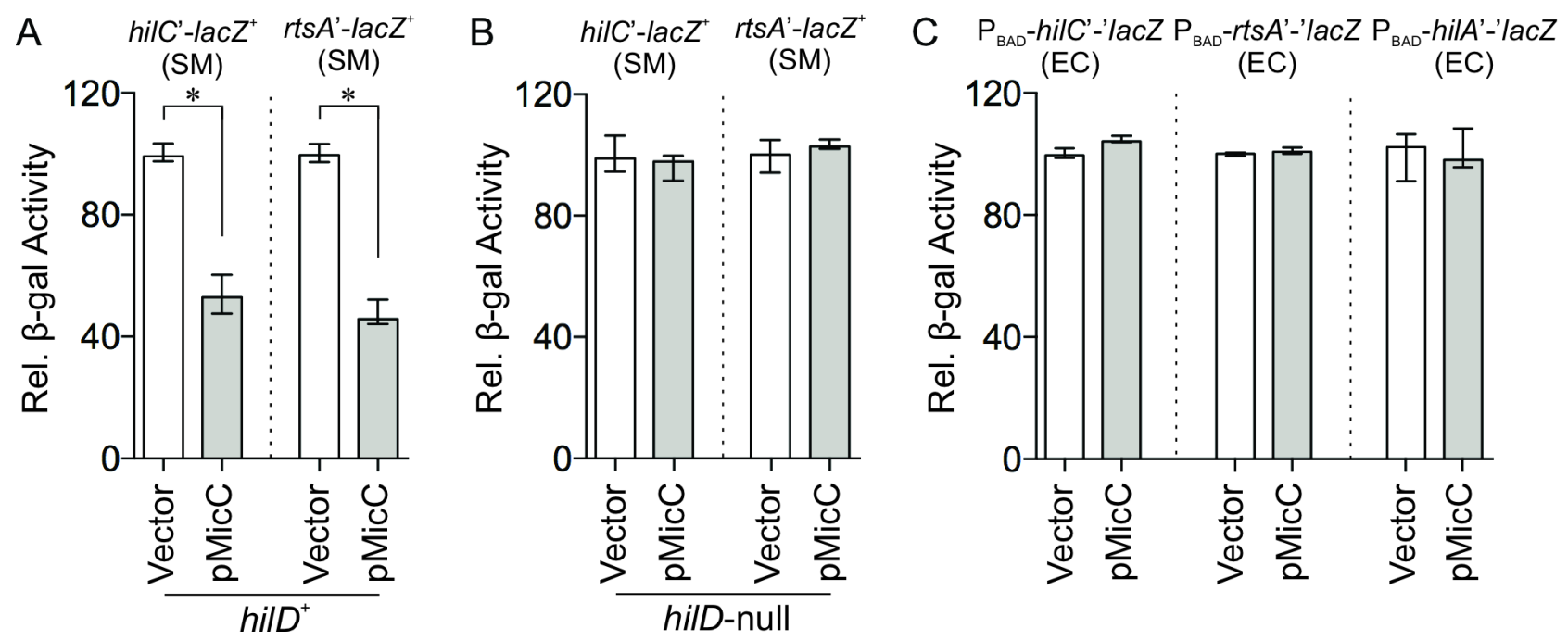

FIG 4. MicC does not regulate HilC, RtsA or HilA. Relative $\beta$-gal activity in Salmonella hilC or hilA transcriptional fusion strains that are (A) hilD+ or (B) hilD-null. (C) Relative $\beta$-gal activity in E. coli hilC''lacZ, rtsA'-'lacZ or hilA'-'lacZ translational fusion strains. All strains include either pBR-plac vector or pMicC plasmid. Results are normalized to each strain containing the vector and are shown as median with interquartile range. Asterisks indicate significant differences between the datasets ( $\mathrm{n}=4, P<$ 0.05, using a Mann-Whitney test). Strains used: JS2187, JS2196, JS2551, JS2552, JMS6503, JMS6504 and JMS6505, with plasmids pBR-plac vector or pMicC. 

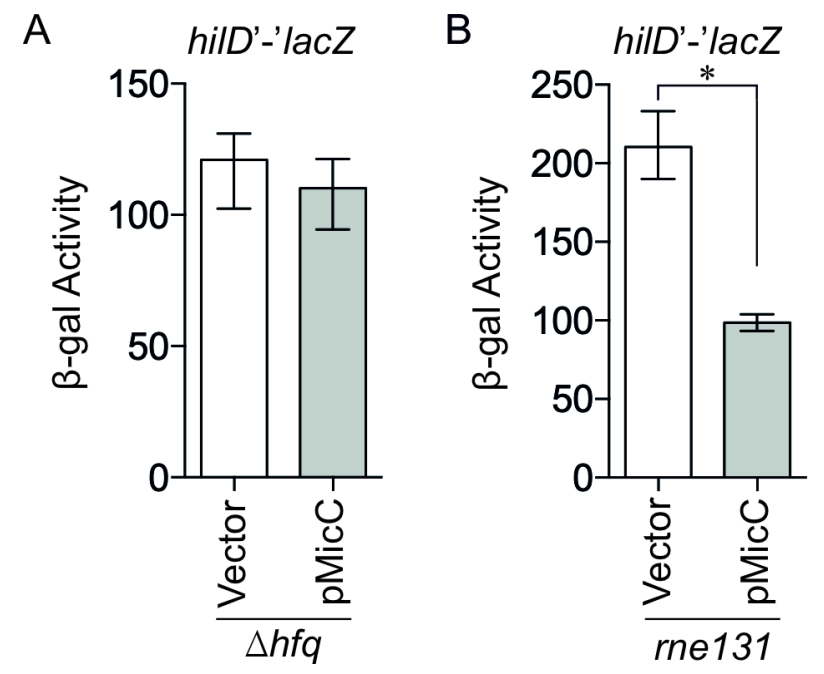

FIG 5. Regulation of hilD mRNA by MicC requires Hfq but not RNAse E. $\beta$-gal activity in Salmonella (A) hfq or (B) rne131 strains harboring the wild type hilD'-'lacZ translational fusion with vector pBRplac or wild type pMicC. Results are shown as median with interquartile range and asterisks indicate significant differences between the datasets ( $n=4, P<0.05$, using a Mann-Whitney test). Strains used: JS2118 and JS2119, with plasmids pBR-plac vector or pMicC. 


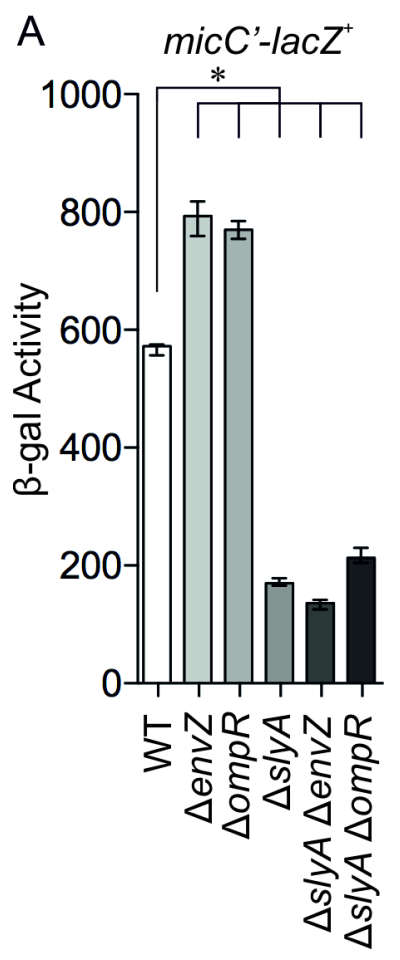

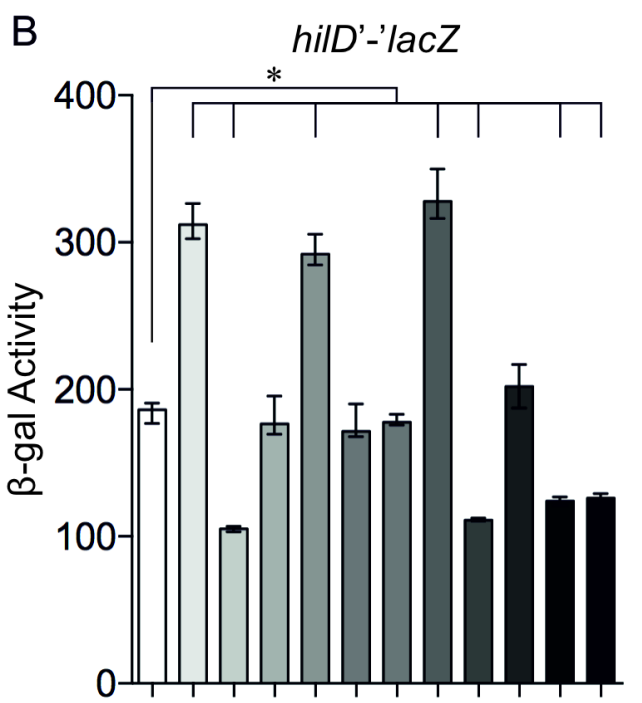

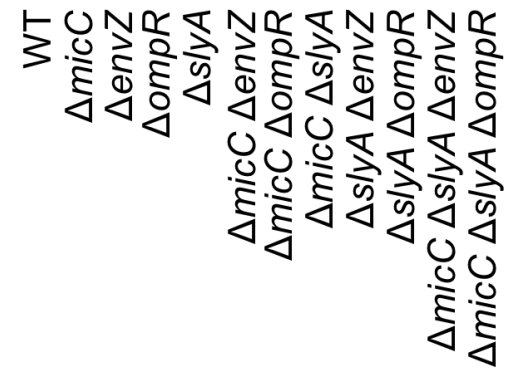

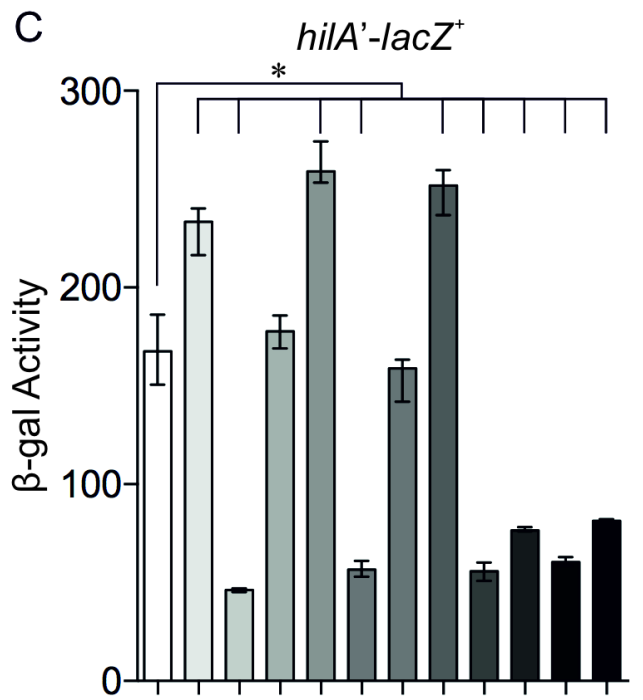

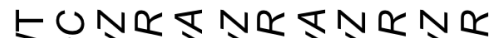
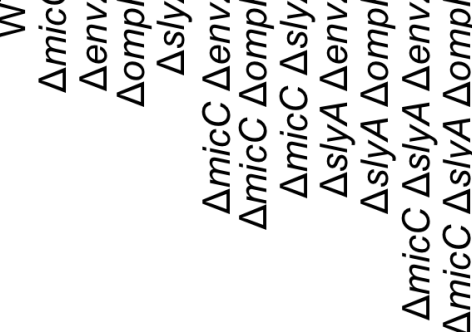

FIG 6. SlyA and EnvZ/OmpR regulate micC and the SPI1 T3SS. $\beta$-gal activity in Salmonella strains

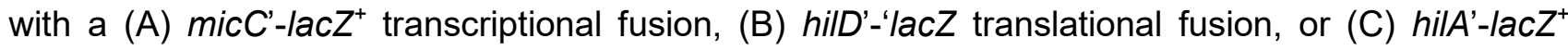
transcriptional fusion in backgrounds containing the indicated mutations. Results are shown as median with interquartile range and asterisks indicate significant differences between the datasets ( $\mathrm{n}$ $\geq 4, P<0.05$, using a Kruskal-Wallis test followed by post hoc Dunn's multiple comparisons). Strains used: JS749, JS892, JS2523, JS2524, JS2525, JS2526, JS2527, JS2528, JS2529, JS2530, JS2531, JS2532, JS2533, JS2534, JS2535, JS2536, JS2537, JS2538, JS2539, JS2540, JS2541, JS2542, JS2543, JS2544, JS2545, JS2546, JS2547, JS2548, JS2549 and JS2550. 
A

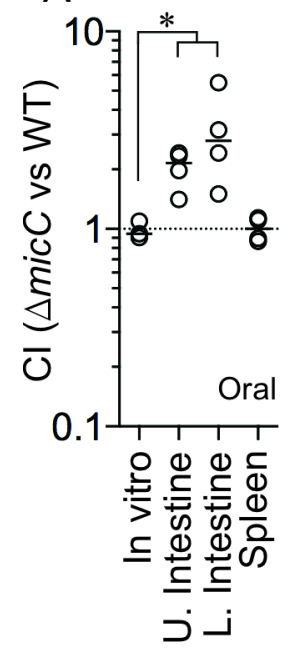

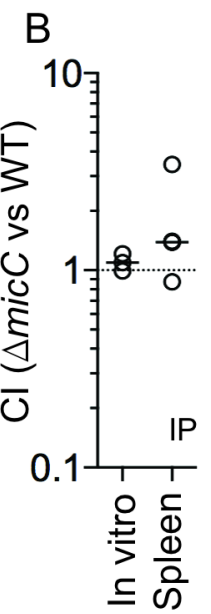

$\mathrm{C}$

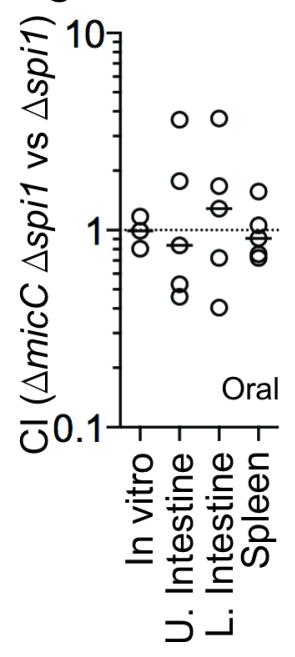

FIG 7. Deletion of MicC provides a fitness advantage in vivo. Competitive index $(\mathrm{Cl})$ for in vitro and in vivo infections comparing the following strains: (A) $\Delta m i c C$ to WT after oral infection, (B) $\Delta m i c C$ to WT after intraperitoneal (IP) infection or (C) $\Delta$ micC $\Delta$ spi1 to $\Delta$ spi1 after oral infection. Upper small intestine (contains duodenum and jejunum), lower small intestine (contains ileum) and spleen were harvested after oral infections, whereas only the spleen was harvested after IP infections. Each circle represents the $\mathrm{Cl}$ from a single mouse. For in vitro competitions, $\mathrm{N}=3$; panel $\mathrm{A}, \mathrm{N}=4$; panels $\mathrm{B}$ and $\mathrm{C}, \mathrm{N}=5$. The horizontal bars indicate the median of each dataset, and the asterisk indicates significant differences $(P<0.05)$ using a Mann-Whitney test. Strains used: JS135, JS2553, JS2554 and JS2555. 